\title{
Efecto de la Estética en la Intención de Compra de Teléfonos Inteligentes
}

\author{
Tarcilla Mariano-Mello(1), Patricio E. Ramírez-Correa ${ }^{(1) \star}$ y Javier Rondan-Cataluña ${ }^{(2)}$ \\ (1) Escuela de Ingeniería, Universidad Católica del Norte, Larrondo 1281, Coquimbo, Chile. \\ (e-mail: tarcilla.mariano@alumnos.ucn.cl; patricio.ramirez@ucn.cl). \\ (2) Departamento de Administración de Empresas y Comercialización e Investigación de Mercados, \\ Universidad de Sevilla, Ramón y Cajal 1, Sevilla, España. (e-mail: rondan@us.es)
}

${ }^{*}$ Autor a quien debe ser dirigida la correspondencia

Recibido Dic. 20, 2017; Aceptado Mar. 5, 2018; Versión final Abr. 2, 2018, Publicado Ago. 2018

\begin{abstract}
Resumen
Se determina el grado de influencia de la apreciación estética sobre la intención de compra de teléfonos inteligentes. Para lograr este propósito se estableció un modelo de investigación basado en la literatura, el cual fue validado usando datos recolectados a través de una encuesta a usuarios de teléfonos inteligentes. Los resultados del análisis indican que si bien la apreciación estética influye directamente en forma moderada sobre la intención de compra de un teléfono inteligente, esta influencia se acentúa en forma indirecta a través de los valores asociados a la compra. Asimismo, los resultados señalan a la forma del teléfono inteligente como la dimensión estética más influyente en la intención de compra. Se concluye que cuando los consumidores aumentan su percepción estética del teléfono inteligente se incrementa su relación emocional con este dispositivo. De la misma forma, su percepción de usabilidad crece, y como consecuencia de esto existe un incremento en la intención de compra.
\end{abstract}

Palabras clave: teléfono inteligente; estética; forma; intención de compra

\section{Effect of Aesthetics on the Purchase Intention of Smartphones}

\begin{abstract}
The degree of influence of the aesthetic appreciation on the purchase intention of smartphones is determined and analyzed. To achieve this purpose, a research model based on literature was established, which was validated using data collected through a survey applied to smartphone users. The results from the analysis indicate that although the aesthetic appreciation directly moderately influences the purchase intention of a smartphone, this influence is indirectly observed through the values associated with the purchase. Likewise, the results indicate that the shape of the smartphone is the most influential aesthetic dimension in the purchase intention. Based on these results, it is concluded that when consumers increase their aesthetic perception of the smartphone their emotional relationship with this device increases. In the same way, their perception of usability increases and, as a consequence of this, there is an increase in the purchase intention.
\end{abstract}

Keywords: smartphones; aesthetic; shape; purchase intention 


\section{INTRODUCCIÓN}

Lee (2014) define teléfono inteligente como un teléfono móvil que ofrece capacidades avanzadas, a menudo con una funcionalidad parecida a la de un computador, y que no se limita a realizar llamadas de voz. En la actualidad el uso de los teléfonos inteligentes es cada día más extenso (ITU, 2016), y debido al deseo de estar en un estado de conexión continua, se ha tornado en un elemento fundamental para las vidas de sus usuarios (Nanda et al., 2016). De hecho, Shin (2012) describe a los teléfonos inteligentes como un artefacto cultural y una extensión del estado social de sus usuarios. Un ejemplo particular de la extensión de estos dispositivos señala que en Brasil y Chile ha aumentado de forma significativa su utilización, llegando el índice de aparatos cada 100 habitantes a 118 y 127, respectivamente (ITU, 2016). Desde la perspectiva académica, el uso de los teléfonos inteligentes se ha analizado ampliamente, desde estudios relacionados con la aceptación de tecnologías (Joo y Sang, 2013; Lin y Kim, 2017), hasta los problemas psicológicos asociados a la dependencia excesiva de ellos (Lee et al., 2014; Van Deursen et al, 2015; Gökçearslan et al., 2016). No obstante lo anterior, existe una carencia en el estudio de los factores físicos que afectan la compra del teléfono inteligente (Toufani et al., 2017).

Dado que normalmente la utilización del teléfono inteligente es observada por otras personas, es un objeto que se asocia al individuo, al igual que puede pasar con la ropa, el calzado o el automóvil. En este tipo de productos el diseño es fundamental para su éxito comercial, así como la estética que es un aspecto clave del diseño (Homburg et al., 2015; Hagtvedt y Patrick, 2014). Por tanto, a medida que un teléfono inteligente obtiene un mayor uso social, la estética del mismo adquiere más peso, posicionándose como un elemento crucial en las ventas de los nuevos modelos. Sin embargo, este aspecto ha sido menos estudiado, especialmente en el contexto latinoamericano. En este contexto, el objetivo de este trabajo es explorar la influencia de la apreciación estética sobre la intención de compra de teléfonos inteligentes. Para ello se examinan dos vías distintas de incluir la apreciación estética en un modelo orientado a explicar la intención de compra, la primera en forma agregada en una variable multidimensional, y la segunda, en forma detallada en relación a los aspectos de la estética. Lazard et al. (2015) define estética como las experiencias sensoriales de placer o belleza resultantes del diseño de una interface. En general, la apariencia o estética del teléfono inteligente es una característica tan importante como el sistema operativo para sus usuarios, pues es la primera cosa que se puede visualizar en ellos, y además, es la primera característica con que el consumidor tiene contacto antes de comprar. Según Swilley (2012), para algunos usuarios el teléfono inteligente es una forma de expresar su estilo de vida, de estar a la moda, de mostrar su individualidad, o hasta de aumentar su estatus. La estética hace que el consumidor perciba las funciones utilitarias y las cualidades del dispositivo como producto (Mishra et al., 2014).

De acuerdo con Toufani et al. (2017), las percepciones estéticas de los usuarios de teléfonos inteligentes se describen en cuatro dimensiones: el diseño, el color, la forma y el tacto, a continuación revisamos cada una de ellas. Según Swilley (2012), el diseño de los teléfonos inteligentes es cada vez más importante, debido que en esta característica recae su atractivo visual, llegando a ser un determinante para el éxito comercial. De hecho, el diseño de un producto puede demostrar su facilidad de la utilización, y por tanto, si un teléfono inteligente posee un diseño complejo, este diseño influye negativamente en su percepción de facilidad de uso, en el caso contrario, un producto con diseño más equilibrado influye positivamente en la demostración de su facilidad de uso (Creusen et al., 2010). Por otra parte, el color de un producto es un elemento simbólico, y a la vez, llama la atención y da estímulo al consumidor, principalmente si buscan variedad en sus opciones de compra (Kauppinen-Räisänen y Luomala, 2010).

En ese sentido, se puede observar que en los productos tecnológicos de consumo, como el teléfono inteligente, esta variedad de colores ha aumentado en los últimos años. La forma de los teléfonos inteligentes ha cambiado de acuerdo con las tendencias sociales, haciendo que los usuarios se diferencien entre sí y ofreciendo un estatus asociado a seguir la moda (Filieri y Lin, 2017). Pero no es solo tendencia, Bar y Neta (2006) señalan que las personas prefieren los productos curvos o aquellos que poseen ángulos redondos, porque ellas utilizan de esas características para predecir si el objeto es seguro o no, de hecho, los ángulos cuadrados y acentuados hacen con que el consumidor asocie al producto con una amenaza. Finalmente, el tacto del teléfono inteligente también ofrece información relevante para el consumidor. Algunos usuarios poseen la necesidad de tocar para adquirir información que no es posible obtener a través de la descripción de los productos o la revisión visual (Peck y Wiggins, 2006). Por ejemplo, si el teléfono inteligente encaja bien en la mano o si es posible contestar rápido al tocarlo. De esta forma, el tacto del teléfono inteligente responde a los estímulos sensoriales del consumidor y puede crear preferencias en su compra (Toufani et al., 2017).

\section{METODOLOGÍA}

La Figura 1 se presenta el modelo conceptual propuesto. En el modelo la estética del teléfono inteligente es una variable latente de segundo orden compuesto por cuadro variables latentes de primer orden: el color, el 
diseño, la forma y el tacto. Asimismo, la estética del teléfono inteligente afecta directamente tanto a la intención de compra de un teléfono inteligente como a los valores que el consumidor asocia con un teléfono inteligente: valor emocional, valor funcional y valor social. Finalmente, el modelo indica que los valores que el consumidor asocia con un teléfono inteligente predicen su intención de compra. Luego, y basados en Toufani et al. (2017), se proponen las siguientes hipótesis: H1) La percepción estética de un teléfono inteligente está relacionada positivamente al valor emocional asociado a un teléfono inteligente; H2) La percepción estética de un teléfono inteligente está relacionada positivamente al valor funcional asociado a un teléfono inteligente; H3) La percepción estética de un teléfono inteligente está relacionada positivamente al valor social asociado a un teléfono inteligente; H4) El valor emocional asociado a un teléfono inteligente está relacionado positivamente con la intención de compra de un teléfono inteligente; H5) El valor funcional asociado a un teléfono inteligente está relacionado positivamente con la intención de compra de un teléfono inteligente; H6) El valor social asociado a un teléfono inteligente está relacionado positivamente con la intención de compra de un teléfono inteligente; y H7) La percepción estética de un teléfono inteligente está relacionada positivamente con la intención de compra de un teléfono inteligente.

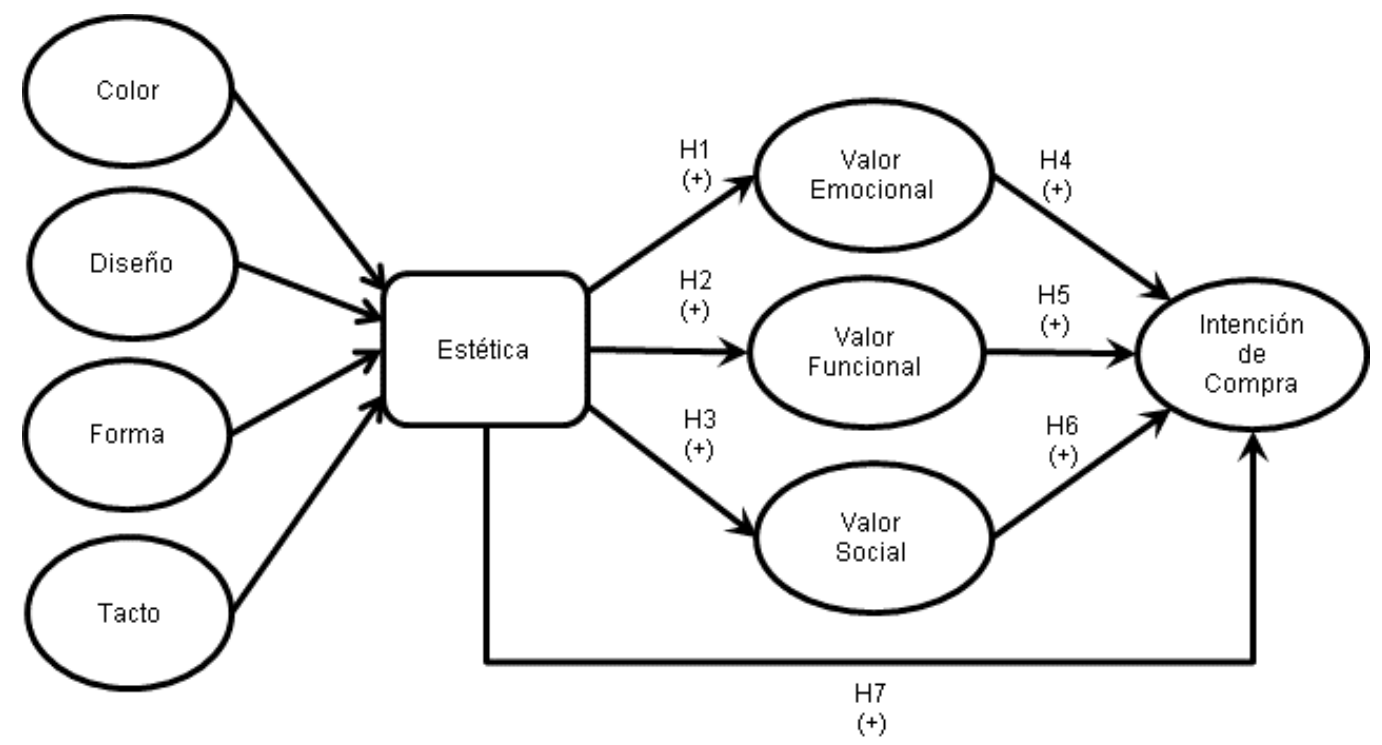

Fig. 1: Modelo Conceptual

El estudio se utilizó una muestra por conveniencia de usuarios de teléfonos inteligentes en Brasil y Chile. Las encuestas fueran aplicadas de través de Internet entre agosto y octubre de 2017 utilizando la herramienta para encuestas LimeSurvey. Con el propósito de hacer operativo el modelo conceptual y testear sus hipótesis, como para explorar las relaciones entre las variables de primer orden que componen a la estética y las demás variables del modelo, se diseñaron dos modelos estructurales. Para analizar estos modelos se utilizó el modelado de ecuaciones estructurales en base a regresiones de mínimos cuadrados parciales (PLS- Partial Least Squares) (Chin, 1998), para estos efectos fue usado el software SmartPLS 3.0. Este estudio tiene dos limitaciones principales: en primer lugar, se ha usado un muestreo no aleatorio, y por tanto, la generalización de los resultados no es posible; y en segundo lugar, dado que los encuestados son de Chile y Brasil, dos culturas latinas, es posible que estos hallazgos difieran en otras culturas.

\section{RESULTADOS Y DISCUSIÓN}

Como muestra la Tabla 1, se obtuvo 388 respuestas válidas, 247 de usuarios brasileños y 141 de usuarios chilenos. De los encuestados, 306 (78,66\%) tenían entre 18 y 29 años, 144 (37,11\%) eran hombres y 238 $(61,34 \%)$ eran mujeres. En relación al sistema operativo, 297 (76,54\%) respondieron que poseían Android y $83(21,39 \%)$ poseían iOS. En su mayoría los usuarios utilizaban teléfonos inteligentes por más de 3 años (42,78\%). La escalas de medida utilizadas fueron tomadas de Toufani et al. (2017) (ver Tabla 2). Todos los indicadores fueron medidos usando una escala tipo Likert de cinco puntos, de "totalmente en desacuerdo" hasta "totalmente de acuerdo". La experiencia en el uso de teléfonos móviles fue medida en años.

Un análisis basado en modelado de ecuaciones estructurales se compone de dos fases, en la primera se valora al modelo de medida y en la segunda el modelo estructural. En la primera fase, y utilizando el procedimiento indicado por Hair et al. (2014) para modelos de componentes jerárquicos, se realizó en análisis para la variable latente de segundo orden Estética. Según este procedimiento, y obedeciendo a las características reflejas de los indicadores de las variables latentes de primer orden que forman la variable Estética (Color, Diseño, Forma y Tacto), en un primer análisis se determinó la fiabilidad individual de indicadores y variables, como también su validez convergente y discriminante. 
Tabla 1: Resultados descriptivos

\begin{tabular}{llrr}
\hline & Categoría & $N$ & $\%$ \\
\hline Género & Femenino & 238 & 61,34 \\
& Masculino & 144 & 37,11 \\
& Otro & 6 & 1,54 \\
\hline Edad & $18-29$ & 306 & 78,86 \\
& $30-39$ & 37 & 9,50 \\
& $40-49$ & 25 & 6,44 \\
& $50-59$ & 12 & 3,09 \\
& 60 o más & 8 & 2,06 \\
\hline Sistema & Android & 297 & 76,54 \\
Operativo & iOs & 83 & 21,39 \\
& Otro & 8 & 2,06 \\
\hline Experiencia & Menos de 1 año & 16 & 4,12 \\
& 1 a 3 años & 66 & 17,01 \\
& 3 a 6 años & 166 & 42,78 \\
& Más de 6 años & 140 & 36,08 \\
\hline Total & & 388 & 100 \\
\hline
\end{tabular}

Tabla 2: Indicadores y cargas de las variables latentes

\begin{tabular}{|c|c|c|c|}
\hline Variable & Código & Carga & Indicador \\
\hline Intención de & IC1 & 0,90 & Es probable que compre mi teléfono inteligente ideal si éste está disponible. \\
\hline \multirow[t]{2}{*}{ Compra } & IC2 & 0,91 & Existe certeza que yo compraré mi teléfono inteligente ideal si éste está disponible. \\
\hline & IC3 & 0,90 & Existe la posibilidad de que yo compre mi teléfono inteligente ideal si está disponible. \\
\hline Valor & VE1 & 0,66 & Me siento emocionado cuando tengo mi teléfono inteligente deseado. \\
\hline \multirow[t]{4}{*}{ Emocional } & VE2 & 0,84 & El uso de mi teléfono inteligente es agradable. \\
\hline & VE3 & 0,73 & Me siento bien cuando mi teléfono inteligente es superior a otros teléfonos inteligentes. \\
\hline & VE4 & 0,82 & Estoy feliz cuando estoy usando mi teléfono inteligente deseado. \\
\hline & VE5 & 0,70 & Siento que mi vida es mejor desde que compré mi teléfono inteligente. \\
\hline \multirow{5}{*}{$\begin{array}{l}\text { Valor } \\
\text { Funcional }\end{array}$} & VF1 & 0,85 & Quiero un teléfono inteligente que tenga una alta fiabilidad. \\
\hline & VF2 & 0,82 & Quiero un teléfono inteligente con un alto grado de funcionalidad. \\
\hline & VF3 & 0,83 & $\begin{array}{l}\text { Quiero un teléfono inteligente que sea útil, basado en sus capacidades técnicas, como } \\
\text { un procesador poderoso que pueda ejecutar diferentes programas al mismo tiempo. }\end{array}$ \\
\hline & VF4 & 0,67 & $\begin{array}{l}\text { Quiero un teléfono inteligente que sea duradero, en términos de su protección contra } \\
\text { daños o en la duración de su batería. }\end{array}$ \\
\hline & VF5 & 0,69 & $\begin{array}{l}\text { Quiero un teléfono inteligente con que posea diversas aplicaciones de software para } \\
\text { distintos propósitos. }\end{array}$ \\
\hline \multirow[t]{2}{*}{$\begin{array}{l}\text { Valor } \\
\text { Social }\end{array}$} & VS1 & 0,86 & $\begin{array}{l}\text { Intento impresionar a mi familia, amigos, o compañeros de trabajo/pares a través de la } \\
\text { compra y uso del teléfono inteligente que deseo. }\end{array}$ \\
\hline & VS2 & 0,86 & $\begin{array}{l}\text { Intento comprar y usar el teléfono inteligente que mi familia, amigos o compañeros de } \\
\text { trabajo seleccionan. }\end{array}$ \\
\hline \multirow[t]{4}{*}{ Color } & E_C1 & 0,75 & Me importa el color de mi teléfono inteligente. \\
\hline & E_C2 & 0,89 & Los teléfonos inteligentes deben venir en diferentes colores. \\
\hline & E_C3 & 0,85 & Deberíamos ser capaces de elegir un teléfono inteligente con varios colores. \\
\hline & E_C4 & 0,73 & Un teléfono inteligente debe tener colores contrastantes que resalten su presencia. \\
\hline \multirow[t]{3}{*}{ Diseño } & E_D1 & 0,81 & $\begin{array}{l}\text { El diseño de mi teléfono inteligente, en base a lo que está disponible como su forma, } \\
\text { tamaño y peso, debe ser único para mí. }\end{array}$ \\
\hline & E_D2 & 0,82 & El diseño de mi teléfono inteligente significa mucho para mí. \\
\hline & E_D3 & 0,76 & El diseño de mi teléfono inteligente debe captar la atención. \\
\hline \multirow[t]{3}{*}{ Forma } & E_F1 & 0,81 & La forma de un teléfono inteligente debe ser agradable a la vista. \\
\hline & E_F2 & 0,88 & Debo disfrutar viendo la forma de mi teléfono inteligente. \\
\hline & E_F3 & 0,83 & La forma de un teléfono inteligente significa mucho para mí. \\
\hline \multirow[t]{4}{*}{ Tacto } & E_T1 & 0,86 & La forma en que se siente mi teléfono inteligente es muy importante para mí. \\
\hline & E_T2 & 0,93 & La textura de mi teléfono inteligente significa mucho para mí. \\
\hline & E_T3 & 0,93 & $\begin{array}{l}\text { La textura de la superficie de mi teléfono inteligente, como su suavidad, es muy } \\
\text { importante para mí. }\end{array}$ \\
\hline & E_T4 & 0,87 & $\begin{array}{l}\text { Las sensaciones transmitidas por mi teléfono inteligente, como la frescura al tacto, son } \\
\text { muy importantes para mí. }\end{array}$ \\
\hline \multirow[t]{4}{*}{ Estética } & Color & 0,76 & Puntuación calculada a partir de los indicadores de Color. \\
\hline & Diseño & 0,86 & Puntuación calculada a partir de los indicadores de Diseño. \\
\hline & Forma & 0,90 & Puntuación calculada a partir de los indicadores de Forma. \\
\hline & Tacto & 0,84 & Puntuación calculada a partir de los indicadores de Tacto. \\
\hline
\end{tabular}


La Tabla 2 muestra las cargas de los indicadores del modelo de medida, siendo todas estas superiores a 0,5. La fiabilidad de las variables se evaluó usando el índice Fiabilidad Compuesta (CR), y la validez convergente se determinó a través de la Varianza Promedio Extraída (AVE), la Tabla 3 muestra estos dos índices, para todas las variables el primero es superior a 0,8 y el segundo mayor a 0,5 . Finalmente, la validez discriminante se evaluó positivamente a través de la comparación de la raíz cuadrada del AVE de cada variable con las correlaciones con las otras variables del modelo, esto se puede ver en la Tabla 4, en ella la diagonal corresponde a la raíz cuadrada del AVE. Probada la bondad del modelo de medida asociado a las variables latentes de primer orden, se procedió a calcular las puntuaciones estandarizadas que las representan en la variable latente de segundo orden. Valorado con éxito el modelo de medida, se procedió a la fase de evaluar los dos modelos estructurales: el Modelo 1 (ver Figura 2), en el que estética es representada como una variable latente de segundo orden de tipo formativo, siendo sus indicadores las puntuaciones estandarizadas de las variables latentes de primer orden asociadas; y el Modelo 2 (ver Figura 3), donde las variables latentes de primer orden asociadas a la estética son representadas directamente en el modelo. La Tabla 5 muestra los índices de ajuste global de los modelos, dado estos valores, se acepta que los modelos se ajustan a los datos, es decir, los datos no contienen más información que la que los modelos transmiten.

La Tabla 6 muestra el resultado del análisis de los modelos estructurales, este resultado señala que la variable dependiente Intención de Compra es explicada un $22 \%$ y un $26 \%$ por el Modelo 1 y el Modelo 2, respectivamente. En el Modelo 1, el coeficiente estandarizado beta asociado a la relación entre Estética e Intención de Compra tiene un valor de 0,11 y es significativo (valor $p<0,05,{ }^{*}$ ). En el Modelo 2, el coeficiente estandarizado beta asociado a la relación entre Forma e Intención de Compra tiene un valor de 0,13 y es significativo (valor $p<0,01,{ }^{* *}$ ). El análisis de la significación de los coeficientes se realizó con la técnica no paramétrica bootstrapping con 5000 sub muestras.

Los resultados del análisis estructural del Modelo 1 permiten apoyar la todas las hipótesis del modelo de investigación. Por otra parte, los resultados asociados al Modelo 2 destacan que la variable Forma tiene el mayor efecto total sobre la variable Intención de Compra, seguida por las variables Diseño y Tacto. Además estas tres variables afectan las percepciones de valor asociadas al teléfono inteligente. De modo contrario, la variable Color no tiene efecto en la Intención de Compra, ni en las percepciones de valor asociadas al teléfono inteligente.

Tabla 3: Coeficientes del modelo de medida

\begin{tabular}{lcc}
\hline Variable & $\begin{array}{c}\text { Fiabilidad } \\
\text { Compuesta (CR) }\end{array}$ & $\begin{array}{c}\text { Varianza Promedio } \\
\text { Extraída (AVE) }\end{array}$ \\
\hline Color & 0,88 & 0,65 \\
Diseño & 0,84 & 0,64 \\
Forma & 0,88 & 0,71 \\
Tacto & 0,94 & 0,81 \\
Estética & 0,91 & 0,70 \\
Intención de Compra & 0,93 & 0,81 \\
Valor Emocional & 0,87 & 0,57 \\
Valor Funcional & 0,88 & 0,60 \\
Valor Social & 0,86 & 0,75 \\
\hline
\end{tabular}

Tabla 4: Análisis de validez discriminante

\begin{tabular}{lccccccccc}
\hline Variable & 1 & 2 & 3 & 4 & 5 & 6 & 7 & 8 & 9 \\
\hline Intención de Compra (1) & $\mathbf{0 , 9 0}$ & & & & & & & & \\
Valor Emocional (2) & 0,42 & $\mathbf{0 , 7 5}$ & & & & & & & \\
Valor Funcional (3) & 0,35 & 0,49 & $\mathbf{0 , 7 8}$ & & & & & & \\
Valor Social (4) & 0,27 & 0,39 & 0,17 & $\mathbf{0 , 8 6}$ & & & & & \\
Estética (5) & 0,35 & 0,58 & 0,38 & 0,37 & $\mathbf{0 , 8 4}$ & & & & \\
Color (6) & 0,18 & 0,35 & 0,24 & 0,19 & nc & $\mathbf{0 , 8 1}$ & & & \\
Diseño (7) & 0,33 & 0,50 & 0,35 & 0,34 & nc & 0,58 & $\mathbf{0 , 8 0}$ & & \\
Forma (8) & 0,36 & 0,57 & 0,37 & 0,34 & nc & 0,56 & 0,67 & $\mathbf{0 , 8 4}$ & \\
Tacto (9) & 0,30 & 0,51 & 0,32 & 0,35 & nc & 0,43 & 0,60 & 0,74 & $\mathbf{0 , 9 0}$ \\
\hline
\end{tabular}




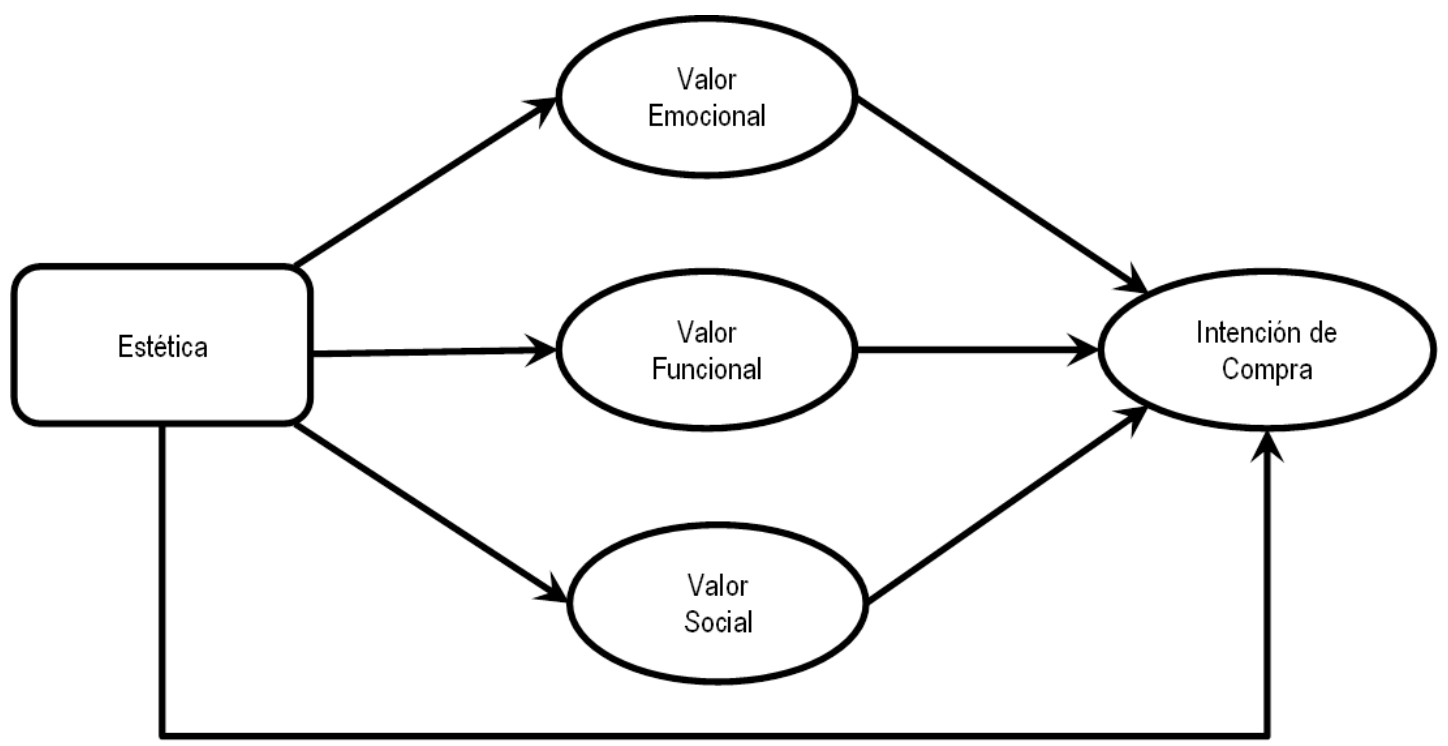

Fig. 2: Modelo 1

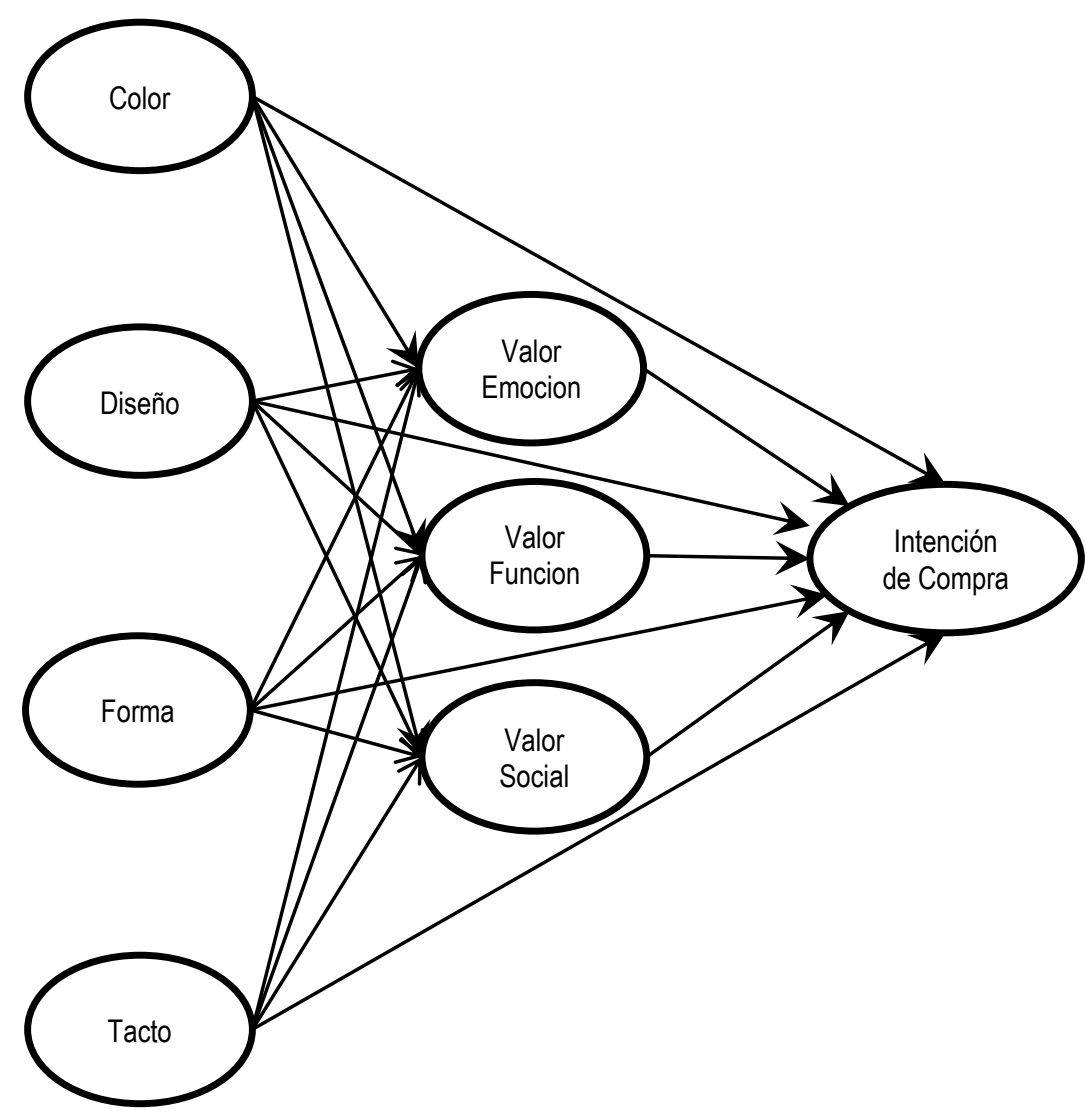

Fig. 3: Modelo 2

Tabla 5: Índices de ajuste del modelo

\begin{tabular}{lcc}
\hline \multicolumn{1}{c}{ Índice } & Modelo 1 & Modelo 2 \\
\hline Coeficiente de trayectoria promedio(APC) & 0,281 & 0,131 \\
$\mathrm{R}^{2}$ promedio (ARS) & 0,215 & 0,238 \\
Factor de inflación de varianza promedio (AVIF) & 1,520 & 2,066 \\
Índice de Ajuste Global (GoF) & 0,384 & 0,406 \\
Raíz cuadrada media residual normalizada (SRMR) & 0,086 & 0,077
\end{tabular}


Tabla 6: Resultados de los modelos estructurales

\begin{tabular}{|c|c|c|c|c|c|c|c|c|c|}
\hline \multirow{2}{*}{$\begin{array}{c}\text { Variable } \\
\text { Dependiente }\end{array}$} & \multirow{2}{*}{$\begin{array}{c}\text { Variable } \\
\text { Independiente }\end{array}$} & \multicolumn{4}{|c|}{ Modelo 1} & \multicolumn{4}{|c|}{ Modelo 2} \\
\hline & & $R^{2}$ & Beta & Sig. & $\begin{array}{c}\text { Efecto } \\
\text { Total }\end{array}$ & $R^{2}$ & Beta & Sig. & $\begin{array}{l}\text { Efecto } \\
\text { Total }\end{array}$ \\
\hline \multirow[t]{9}{*}{ Intención de Compra } & & 0,22 & & & & 0,26 & & & \\
\hline & Valor Emocional & & 0,22 & * & 0,22 & & 0,21 & $* \star *$ & 0,21 \\
\hline & Valor Funcional & & 0,18 & $* \star *$ & 0,18 & & 0,17 & *** & 0,17 \\
\hline & Valor Social & & 0,11 & $* \star *$ & 0,11 & & 0,10 & * & 0,10 \\
\hline & Estética & & 0,11 & * & 0,35 & & & & \\
\hline & Color & & & & & & 0,01 & ns & 0,00 \\
\hline & Forma & & & & & & 0,13 & ** & 0,25 \\
\hline & Tacto & & & & & & 0,04 & ns & 0,11 \\
\hline & Diseño & & & & & & 0,08 & ns & 0,15 \\
\hline \multirow[t]{6}{*}{ Valor Emocional } & & 0,35 & & & & 0,34 & & & \\
\hline & Estética & & 0,59 & $* * *$ & & & & & \\
\hline & Color & & & & & & $-0,04$ & ns & \\
\hline & Forma & & & & & & 0,30 & $* * *$ & \\
\hline & Tacto & & & & & & 0,20 & $* * *$ & \\
\hline & Diseño & & & & & & 0,16 & $* * *$ & \\
\hline \multirow[t]{6}{*}{ Valor Funcional } & & 0,15 & & & & 0,17 & & & \\
\hline & Estética & & 0,38 & $* \star \star$ & & & & & \\
\hline & Color & & & & & & $-0,04$ & ns & \\
\hline & Forma & & & & & & 0,27 & $* * *$ & \\
\hline & Tacto & & & & & & 0,07 & ns & \\
\hline & Diseño & & & & & & 0,13 & ** & \\
\hline \multirow[t]{6}{*}{ Valor Social } & & 0,14 & & & & 0,18 & & & \\
\hline & Estética & & 0,37 & $* * *$ & & & & & \\
\hline & Color & & & & & & 0,06 & ns & \\
\hline & Forma & & & & & & 0,10 & * & \\
\hline & Tacto & & & & & & 0,17 & $\star \star *$ & \\
\hline & Diseño & & & & & & 0,22 & $* * *$ & \\
\hline
\end{tabular}

A partir de los resultados se desean resaltar seis ideas. Primero, al igual que los resultados de Toufani et al. (2017), la estética explica en alto grado el valor emocional, y por tanto, las emociones de los consumidores en relación con el teléfono inteligente están influenciadas por las percepciones que los consumidores poseen sobre la estética. De esa forma, cuanto mayor es la percepción de la estética del teléfono inteligente, los consumidores van tener una mayor relación emocional con este producto. Segundo, distinto a los resultados de Toufani et al. (2017), este estudio encontró que la intención de compra de un teléfono inteligente es explicada por el valor funcional. Este resultado podría relacionarse con la mayor disponibilidad de tecnologías de última generación en la muestra original de usuarios australianos y la menor disponibilidad de estas tecnologías en la muestra de este estudio.

Tercero, que la estética del teléfono inteligente explique su intención de compra apoya los hallazgos de Nanda et al. (2008), en el sentido de que la estética es uno de los factores más importantes para los consumidores de teléfonos inteligentes cuando deciden comprar este producto. Y por lo tanto, si el teléfono inteligente posee una estética agradable para el consumidor, la percepción de usabilidad es mayor y, consecuentemente, la intención de compra aumenta. Cuarto, la forma es la única dimensión de la estética que explica directamente la intención de compra del teléfono inteligente. Este resultado es apoyado por el estudio de Filieri y Lin (2017), que señala que los diseñadores combinan elementos visuales, como la forma, para atraer los consumidores a una mayor interacción con el producto y, consecuentemente, considerar la compra.

Quinto, al igual que en Toufani et al. (2017), la intención de compra es explicada por el valor social del teléfono inteligente, pero en el actual estudio esta variable tiene un efecto menor. Este resultado es consistente con Hofstede (2017), pues en la comparación cultural de la dimensión individualismo, Australia supera a Brasil y Chile. Es decir, al pertenecer los encuestados a sociedades más colectivistas existen menores motivaciones de diferenciarse de los otros a través de la compra de un teléfono inteligente. Sexto y último, centrándonos en los aspectos incluidos dentro de la estética (color, forma, tacto y diseño), llama la atención el hecho de que el color no es un aspecto importante, ya no solo en la compra de un teléfono móvil, sino que tampoco en el valor emocional, funcional o social. Esto indica que este atributo del producto no tiene ningún efecto entre las personas encuestadas en el estudio. Teniendo en cuenta que para los humanos el sentido de la vista es el primordial y que los colores afectan a muchas de nuestras percepciones, resulta interesante el hecho de que para este tipo de productos parezca no tener ningún efecto. 
Finalmente, se enfatizan algunos elementos importantes que nacen del análisis comparativo entre los resultados del estudio y los antecedentes encontrados en la literatura. Desde una perspectiva general y en relación al efecto de la estética en los valores asociados a un producto, los hallazgos del estudio se alinean tanto al trabajo de Hagtvedt (2011), quien basado en una muestra de consumidores predominantemente Estadounidenses reporta que la forma del logotipo de un producto afecta a la percepción de innovación y a la confianza del consumidor, como al de Mishra (2016), quien desde India informa que existe una fuerte relación entre la percepción de diseño y el valor de marca de un producto. Desde la perspectiva del efecto positivo de la estética en la aceptación del teléfono inteligente, los resultados de este trabajo apoyan lo reportado por Shin (2012), quien indica una relación entre la estética percibida y la actitud hacia los teléfonos inteligentes en una muestra de usuarios de Estados Unidos y Corea. Asimismo, es posible apoyar con estos hallazgos los resultados de Kim et al. (2017), quienes indican que entre los consumidores coreanos la estética afecta positivamente el valor percibido de un producto aumentado. Estos autores consideran que un producto aumentado es un producto que tiene los atributos físicos principales (por ejemplo un teléfono inteligente) y los atributos no físicos que se agregan para aumentar su valor (por ejemplo los accesorios del teléfono inteligente).

En relación a los efectos de las dimensiones color y tacto de la estética del teléfono inteligente, es posible reportar una mixtura en la concordancia de los resultados de este estudio con los hallazgos de otras investigaciones. Por una parte, el nulo efecto del color, tanto en los valores asociados a la compra del teléfono inteligente como en la intención de compra, se distancia de los resultados señalados por autores como Wedel y Pieters (2014), quienes indican que el color de un producto afecta la percepción de la marca, o de Elliot y Maier (2014), quienes señalan que el color de un producto se asocia a la evocación de emociones y pensamientos. Por otra parte, el nulo efecto del tacto en el valor funcional del teléfono inteligente ratifica los resultados de Sonderegger y Sauer (2015), quienes utilizando una muestra de estudiantes suizos concluyen que la estética no visual no contribuye a aumentar la valoración de usabilidad de un producto, y adicionalmente, el resultado asociado al efecto de tacto en los valores emocional y social del teléfono inteligente, abre un nuevo camino de exploración de la incidencia de la estética no visual en la valorización de un producto.

\section{CONCLUSIONES}

Los resultados del estudio permiten concluir que cuando los consumidores aumentan su percepción estética del teléfono inteligente se incrementa su relación emocional con este dispositivo, y de la misma forma, su percepción de usabilidad crece, y como consecuencia de esto, existe un incremento en su intención de compra. Por otra parte, el análisis muestra que la forma del teléfono inteligente es la dimensión estética más influyente en la intención de compra, y por tanto, se propone como la característica más relevante al momento de diseñar campañas de publicidad de este tipo de dispositivos en el mercado de Brasil y Chile.

\section{REFERENCIAS}

Bar, M. y M. Neta, Humans prefer curved visual objects, doi: 10.1111/j.1467-9280.2006.01759.x, Psychol. Sci., 17(8), 645648 (2006)

Chae, M. y J. Kim, Do size and structure matter to mobile users? An empirical study of the effects of screen size, information structure, and task complexity on user activities with standard web phones, doi:10.1080/01449290410001669923 Behav. Infor. Technol., 23(3), 165-181(2004)

Creusen, M.E., R.W. Veryzer y J.P. Schoormans, Product value importance and consumer preference for visual complexity and symmetry, doi:10.1108/03090561011062916, Eur. J. Mark., 44(9/10), 1437-1452 (2010)

Elliot, A.J. y M.A. Maier, Color psychology: Effects of perceiving color on psychological functioning in humans, doi: 10.1146/annurev-psych-010213-115035, Annu. Rev. Psychol., 65, 95-120 (2014)

Filieri, R. y Z. Lin, The role of aesthetic, cultural, utilitarian and branding factors in young Chinese consumers' repurchase intention of smartphone brands, doi:10.1016/j.chb.2016.09.057, Comput. Hum. Behav., 67, 139-150 (2017)

Gökçearslan, Ş., F. K. Mumcu, T. Haşlaman y Y. D. Çevik, Modelling smartphone addiction: The role of smartphone usage, self-regulation, general self-efficacy and cyberloafing in university students, doi:10.1016/j.chb.2016.05.091, Comput. Hum. Behav., 63, 639-649 (2016)

Hagtvedt, H. y V. M. Patrick, Consumer response to overstyling: Balancing aesthetics and functionality in product design, doi:10.1002/mar.20713, Psychol. \& Mark, 31(7), 518-525 (2014)

Hagtvedt, H., The impact of incomplete typeface logos on perceptions of the firm, doi:10.1509/jmkg.75.4.86, J. Mark., 75(4), 86-93 (2011)

Hair, J. F., M. Sarstedt, L. Hopkins y V.G. Kuppelwieser, Partial least squares structural equation modeling (PLS-SEM), doi:10.1108/EBR-10-2013-0128, Eur. Bus. Rev., 26(2), 106-121 (2014) 
Hofstede, G. H., The Hofstede Insights Culture Compass, Acceso: 15 de Octubre (2017)

Homburg, C., M. Schwemmle y C. Kuehnl, New product design: Concept, measurement, and consequences, doi:10.1509/jm.14.0199, J. Mark., 79(3), 41-56 (2015)

ITU, ICT statistics, Acceso: 8 de Diciembre (2016)

Joo, J. y Y. Sang, Exploring Koreans' smartphone usage: An integrated model of the technology acceptance model and uses and gratifications theory, doi:10.1016/j.chb.2013.06.002, Comput. Hum. Behav., 29(6), 2512-2518 (2013)

Kauppinen-Räisänen, H. y H. T. Luomala, Exploring consumers' product-specific colour meanings, doi: 10.1108/13522751011053644, Qual. Mark. Res., 13(3), 287-308 (2010)

Kim, S.J., K.H. Kim y J. Choi, The role of design innovation in understanding purchase behavior of augmented products, doi:10.1016/j.jbusres.2017.09.047, J. Bus. Res., en prensa (2017)

Lazard, A. J., I. Watkins, M.S. Mackert, B. Xie, K. K. Stephens y H. Shalev, Design simplicity influences patient portal use: the role of aesthetic evaluations for technology acceptance, doi: 10.1093/jamia/ocv174, J. Am. Med. Inform. Assoc., 23(1), 157-161 (2015)

Lee, S.Y., Examining the factors that influence early adopters'smartphone adoption: The case of college students, doi:10.1016/j.tele.2013.06.001, Telemat. Inform., 31(2), 308-318 (2014)

Lee, Y.K., C.T. Chang, Y. Lin y Z. H. Cheng, The dark side of smartphone usage: Psychological traits, compulsive behavior and technostress, doi:10.1016/j.chb.2013.10.047, Comput. Hum. Behav., 31, 373-383 (2014)

Lin, F. y T. Kim, Examining the Determinants of Smartphone User's User Satisfaction and Intention to Use Social Networking Service with TAM, Transylv. Rev., 1(3), 1-12 (2017)

Mishra, A., Attribute-based design perceptions and consumer-brand relationship: Role of user expertise, doi:10.1016/j.jbusres.2016.05.012, J. Bus. Res., 69(12), 5983-5992 (2016)

Mishra, A., S. B. Dash y D. Cyr, Linking user experience and consumer-based brand equity: the moderating role of consumer expertise and lifestyle, doi:10.1108/JPBM-12-2013-0459, J. Prod. Brand Manage., 23(4/5), 333-348 (2014)

Nanda, P., J. Bos, K.L. Kramer, C. Hay y J. Ignacz, Effect of smartphone aesthetic design on users' emotional reaction: An empirical study, doi:10.1108/17542730810881339, TQM J., 20(4), 348-355 (2008)

Peck, J. y J. Wiggins, It just feels good: Customers' affective response to touch and its influence on persuasion, doi:10.1509/jmkg.70.4.56, J. Mark., 70(4), 56-69 (2006)

Shin, D., Cross-analysis of usability and aesthetic in smart devices: what influences users'preferences?, doi:10.1108/13527601211270020, Cross Cult. Manag.,19(4), 563-587 (2012)

Sonderegger, A. y J. Sauer, The role of non-visual aesthetics in consumer product evaluation, doi: 10.1016/j.ijhcs.2015.05.011, Int. J. Hum. Comput. Stud., 84, 19-32 (2015)

Swilley, E., Aesthetic technology: scale development and measurement, doi:10.1504/IJTMKT.2012.048119, Int. J. Technol. Mark., 7(3), 324-341 (2012)

Toufani, S., J.P. Stanton y T. Chikweche, The importance of aesthetics on customers' intentions to purchase smartphones, doi:10.1108/MIP-12-2015-0230, Mark. Intell. Plan., 35(3), 316-338 (2017)

Van Deursen, A. J., C. L. Bolle, S. M. Hegner y P. A. Kommers, Modeling habitual and addictive smartphone behavior: The role of smartphone usage types, emotional intelligence, social stress, self-regulation, age, and gender, doi:10.1016/j.chb.2014.12.039, Comput. Hum. Behav., 45, 411-420 (2015)

Wedel, M. y R. Pieters, The buffer effect: The role of color when advertising exposures are brief and blurred, doi: 10.1287/mksc.2014.0882, Mark. Sci., 34(1), 134-143 (2014) 
